\section{RESULTS}

As shown in Fig. 1, when given no food Ss drank more saline than water in both singleand double-bottle tests. With food given Ss still drank more saline with a single bottle, but with double bottles they switched to water. For single-bottle data, analysis of variance shows a statistically significant difference $(p<.05)$ for both food/no-food and saline/water comparisons. The slight nonparallelism between the saline and water curves (interaction) is not significant $(p>.05)$. For the double-bottle data, analysis shows significant interaction effect with both main effects statistically not significant.

\section{DISCUSSION}

The results clearly show that the preference for isotonic saline $(0.9 \%)$ depends upon the water need induced by dry-food ingestion. The saline curve and water curve with a single bottle are statistically parallel but, using the same Ss as their own control, Hsiao $(1967)^{2}$ showed a steeper increase in water intake from no-food to food conditions than the comparable increase in $0.9 \%$ saline or in $1.8 \%$ saline in take.

The preference change with double bottles cannot be due to adaptation to saline taste. In his unpublished data, Hsiao found that even in the first hour rats preferred water to saline when they ingested dry food.

Bare (1949), using a 24h double-bottle test, showed that rats drink more hypotonic or isotonic saline solutions than water when food is available ad lib. However, about $10 \mathrm{cc}$ of water is drunk by rats in addition to the saline solutions. Rats may drink more water while eating food but drink more saline when they are not eating.

Stricker \& Wolf (1967) reported that even after injection of strong hypertonic saline rats drink more hypertonic saline than water in a double-bottle test. Comparing the results obtained by Deutsch \& Jones (1960), Falk \& Titlebaum (1963) and those cited above with the present results it should be noted that water is preferred to isotonic saline in a double-bottle test only when the rat is hungry and is taking dry food meal. Since rats eat less food when given $0.9 \%$ saline than water (Hsiao, 1967) they may have chosen water in order to eat more food.

\section{REFERENCES}

BARE, J. K. The specific hunger for sodium chloride in normal and adrenalectomized white rats. Journal of Comparative \& Physiological Psychology, 1949, 42, 242-253.

DEUTSCH, J. A., \& JONES, A. D. Diluted water: An explanation of the rat's preference for saline. Journal of Comparative \& Physiological Psychology, 1960,53, 122-127.

FALK, J.L., \& TITLEBAUM, L.F. Saline solution preference in the rat: Further demonstrations.

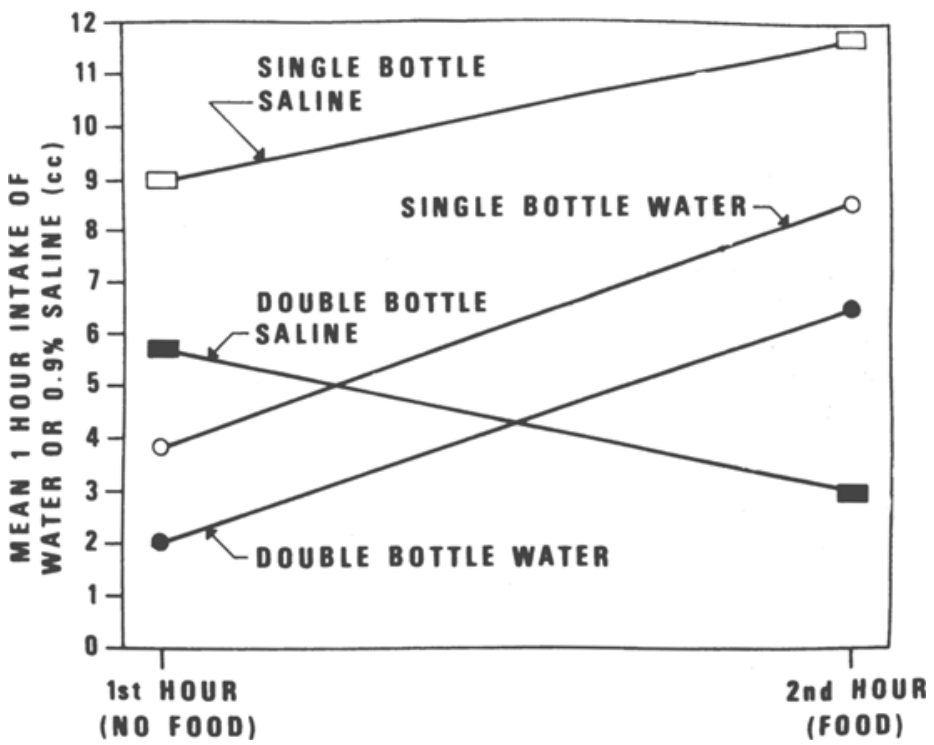

Fig. 1. One-hour intake of water or $0.9 \%$ saline solution in singleand double-bottle tests with or without food available in 24-h food/water-deprived rats.

Journal of Comparative \& Physiological Psychology, 1963, 56, 337-342.

HSIAO, S. Saline drinking effects on food and water intake in rats. Psychological Reports, $1967,21,1025-1028$

O'KELLY, L. I. The effect of preloads of water and sodium chloride on voluntary water of thirsty rats. Journal of Comparative \& Phy siological Psychology, 1954, 47, 7-13.

LEPKOVSKY, S., LYMAN, R., FLEMING, D.

NAGUMO, N., \& DIMICK, M. M. Gastrointestinal regulation of water and its effect on food intake and rate of digestion. American Journal of Physiology, 1957, 188, 327-331.

RICHTER, C. P. Salt appetite of mammals: It dependence on instinct and metabolism. In $L$ 'instinct dans le comportement des animaux et de l'homme. Paris: Masson, 1956. Pp. 577-632. STELLAR, E., HYMAN, R., \& SAMET, S., Gastric factors controlling water- and salt-solution drinking. Journal of Comparative \& Physiological Psychology, 1954, 47, 220-226.

STRICKER, E. M., \& WOLF, G. Hypovolemic thirst in comparison with thirst induced by hyperosmolarity. Physiology \& Behavior, 1967 , 2,33-37.

WEINER, I.H., \& STELLAR, E. Salt preference of the rat determined by a single-stimulus method. Journal of Comparative \& Physiological Psychology, 1951, 44, 394-401.

\section{NOTES}

1. This study was supported by PHS Research Grant MH 15530-01. We thank Eldonna Fisher for editorial and secretarial aid.

2. Also, Hsiao, S. Salted food and saline solutions intake by rats. Submitted to Journal of Comparative \& Physiological Psy chology, 1969.

\title{
Impaired acquisition of a successive visual pattern discrimination following hippocampal ablations in cats
}

\section{A. A. BUERGER, ${ }^{2}$ Department of Physical Biology and Section of Neurobiology and Behavior, Comell University, Ithaca, N.Y. 14850}

Three groups of mature cats were tested for acquisition of a successive visual pattern discrimination. Ammon's horn and pyriform cortex of Group $1(N=6)$ had been ablated. Group $2(N=4)$ were shamoperated and Group $3(N=3)$ were intact normal animals. Group 1 was significantly inferior to the combined control groups $(p<0.004)$. Hence, ablation of the hippocampal region severely impairs the acquisition of successive discriminations in cats.

The literature suggests that lesions of the hippocampal region produce postoperative acquisition deficits that are more severe for successive than for simultaneous problems. Kimble (1963) found that hippocampal rats were impaired on a successive, but not on a simultaneous, brightness discrimination. Stein \& Kimble (1966) also found that hippocampectomized rats were not 
impaired on the acquisition of a simultaneous brightness discrimination. Kimble and Stein's findings are also supported by those of Niki (1965) who found that hippocampal rats were impaired in the acquisition of a brightness discrimination in a continuously reinforced operant conditioning situation. Such a situation may be viewed as a successive brightness discrimination without an intertrial interval. In previous studies of cats, hippocampal lesions produced no deficits in the learning of simultaneous pattern discriminations (Teitelbaum, 1964; Webster \& Voneida, 1964). The present study attempted to discover if lesions of the hippocampal region in the cat would produce deficits in the acquisition of a successive visual pattern discrimination.

\section{METHODS}

The acquisition of a successive visual pattern discrimination was compared in hippocampal, sham-operated, and normal cats. Thirteen adult cats were divided into three groups. The six Ss in Group 1 had bilateral hippocampal ablations; the four Ss in Group 2 had bilateral sham operations; three intact cats formed Group 3. No animals were trained on any pattern discrimination prior to surgery; however, all animals had learned auditory and brightness discriminations (Buerger, 1967). The apparatus and procedure in these auditory and brightness discriminations were identical to those described below except that a $300-\mathrm{Hz}$ tone was substituted for the horizontal stimulus, and a lighted square was substituted for the vertical stimulus.

All surgery was performed in one step under pentobarbital anesthesia. The pyriform cortex, the ventral portions of Ammon's horn, and a small part (5-10 $\left.\mathrm{mm}^{3}\right)$ of the temporal region dorsolateral to the pyriform cortex were aspirated from Group 1. Only that same small part of the temporal region was removed from Group 2. Group 3 was unoperated. The surgical technique and the chamber used in this experiment have been described elsewhere (Buerger, 1967).

The Ss learned a successive visual pattern discrimination. The stimuli were three horizontal bars $(\mathrm{H})$ or three vertical bars $(\mathrm{V})$ of equal brightness presented randomly on a translucent tapping key for $6 \mathrm{sec}$. The Ss were fed condensed milk from a solenoiddriven dipper if the key was pressed when $V$ was presented or if the key was not pressed when $H$ was presented. The $S s$ were shocked through a floor grid if they pressed when $\mathbf{H}$ was presented or did not press when $V$ was presented. There was a $3-\sec$ rest period between consecutive stimuli. Each day, Ss were allowed 50 correct responses and as many errors as they required to reach this criterion. Ss were run every day until they reached a criterion of six or less errors on 3 consecutive days. However, no $S$ was run more than 40 days. All Ss were maintained at $80-85 \%$ of their ad lib weight by feeding them commercial cat food and/or vitamin pills. The Mann-Whitney test (Siegel, 1956) was used to evaluate the behavioral data.

All animals were sacrificed with pentobarbital and perfused with $10 \%$ formalin. Brains were cut into 3- to 5-mm blocks and photographed. Representative sections from these blocks were stained with Luxol fast blue, cresyl violet, and hematoxylin and eosine. ${ }^{3}$

\section{RESULTS}

The Ss in Group 1 showed no surgical damage to structures adjacent to the hippocampal region such as the thalamus and optic radiation. Group 2 showed no hippocampal lesions and Group 3 appeared normal. Dorsal portions of Ammon's horn were present in all of Group 1. Two Ss in Group 1 also had small segments of the anterior ventral tip of Ammon's horn remaining. However, in all cases this remnant was isolated from the dorsal portions. The pyriform cortex was removed from all of Group 1.

The Ss in Group 1 required 40t, 40+, 34, 22,19 , and 15 days to reach criterion. The Ss in Group 2 required 14,11,9, and 9 days, and the normal animals (Group 3) took 30, 8 , and 5 days. The hippocampal Ss were significantly inferior to the combined control groups ( $p<0.004)$. Also, Group 1 was impaired in comparison with the sham Ss $(p<0.005)$. However, the normal group was not significantly different from the hippocampal Ss $(p<0.083)$. This fact was clearly due to the normal $S$ which took 30 days to reach criterion. Finally, the normal and sham groups were not significantly different $(p<0.20)$; the same normal animal mentioned above may be responsible also for the insignificance of this difference.

\section{DISCUSSION}

The results of this experiment clearly show that, in the cat, lesions of the ventral hippocampus (Ammon's horn) and/or pyriform cortex impair the acquisition of a successive visual discrimination. Hence, the cat and rat seem similar in this regard. It would be of interest to discover the reasons for the clear separation between the sham-operated and hippocampal groups. There are at least two general classes of explanation: anatomical and behavioral. The lesions in the experimental group included both pyriform cortex and ventral hippocampus. They are similar to the ablations which are reported to reduce the ability of neurosurgical patients to learn new information (Barbizet, 1963). It may be that production of a deficit in acquisition of discriminations by cats requires lesions of both pyriform cortex and hippocampus. However, it is probable that the type of learning situation used in this experiment was a more important determinant. In addition to the reports cited in the introduction, Orbach, Milner, \& Rasmussen (1960) found no evidence of impairment of simultaneous pattern and object discriminations in monkeys. Hence, the bulk of the evidence may indicate that ablations of the hippocampal region disrupt acquisition of successive discriminations more than simultaneous discriminations. It would be interesting to know if hippocampal lesions in other animals, such as monkeys, produce similar deficits on successive pattern discriminations.

\section{REFERENCES}

J. Defect in memorizing of hippocampal-mammillary origin: A review. Joumal of Neurology, Neurosurgery \& Psy chiatry, 1963, 26, 127-135.

BUERGER, A. A. The effects of hippocampal and pyriform ablations on the formation of memory in the cat. Cognitive Sy stems Research Program, Report No. 11, 1-49.

KIMBLE, D. P. The effects of bilateral hippocampal lesions in rats. Journal of Comparative \& Physiological Psychology, 1963, 56, $273-283$.

NIKI, $H$. The effect of hippocampal ablation on the inhibitory control of operant behavior in the rat. Japanese Psychological Research, 1965, 7, 126-137.

ORBACH, J., MILNER, B., \& RASMUSSEN, T. Learning and retention in monkeys after amygdala-hippocampal resection. A.M.A. Archives of Neurology, 1960, 230-251.

SIEGEL, S. Nonparametric statistics. New York: McGraw-Hill, 1956.

STEIN, D. G., \& KIMBLE, D. P. Effects of hippocampal lesions and post-trial strychnine administration on maze behavior in the rat. Joumal of Comparative \& Physiological Psychology, 1966, 62, 243-249.

TEITELBAUM, H. A comparison of effects of orbitofrontal and hippocampal lesions upon discrimination learning and reversal in the cat. Experimental Neurology, 1964,9, 452-462.

WEBSTER, D. B., \& VONEIDA, T. J. Learning deficits following hippocampal lesions in split-brain cats. Experimental Neurology, 1964, $10,170-182$.

1. This research was partially supported by funds under United States Public Health Service Grants NIG No. NB-05381 and No. 5TI-GM-223 and Office of Naval Research Contract No. NONR $401(40)$. The preparation of this manuscript was supported in part by Grant No. MH-14471 from the National Institute of Mental Health to C. G. Gross, and in part by NIH Postdoctoral Fellowship No. 1-F2-MH-40,532-01 given to the author. I thank Professors F. Rosenblatt and E. L. Gasteiger for their advice and support. The manuscript was prepared while the author was a Postdoctoral Fellow at the Department of Psychology, Harvard University. I thank the faculty and staff of that department.

2. Present address: Department of Psychology, Harvard University, Cambridge, Mass. 02138.

3. I wish to thank Professor J.H. Ferguson and C. Poole of the Department of Pathology, Upstate Medical Center, State University of New York for sectioning and staining these slides. 